\title{
Erläuterungen zur Edition
}

Die Edition bietet die vollständigen Texte aller nach gegenwärtigem Kenntnisstand überlieferten Briefe oder Brieffragmente von und an Johann Christoph Gottsched und Luise Adelgunde Victorie Gottsched, geborene Kulmus. Als Brief wird jeder nichtfiktionale Text verstanden, der von einem Absender an einen Empfänger, sei es eine Person oder eine Personengruppe, gerichtet ist und nach der Intention des Autors nicht für eine Veröffentlichung vorgesehen war. Einen Grenzfall bilden Zuschriften, die Gottsched als Zeitschriftenherausgeber empfangen hat, die also durchaus für eine eventuelle Publikation gedacht waren. Besitzen diese Schreiben formal den Charakter eines Briefes, also eine Anrede, Grußformeln und Datum, haben wir uns für die Aufnahme in die Ausgabe entschieden. Die Leipziger Sammlung von Briefen an Gottsched enthält einige Schreiben, die der Kasuallyrik zuzurechnen sind. Sofern sie durch Anrede und/oder Unterschrift, Datum und Ort formale Charakteristika der Gattung Brief aufweisen, werden sie in unserer Ausgabe mitgeteilt. Handelt es sich hingegen um Gedichte ohne diese formalen Kriterien, verzichten wir auf den Abdruck, auch wenn die Texte von Wolfgang Suchier ${ }^{1}$ unter den Korrespondenzen aufgeführt sind. So werden Gedichte Franz Christoph von Scheybs vom 1. bzw. 6. Oktober 1749 (Suchier S. 65) nicht wiedergegeben, da sie schwerlich als Briefe anzusehen sind, während andere Texte in Versform aus inhaltlichen Gründen aufgenommen wurden (vgl. Nr. 31).

Briefbeilagen, seien es Fremdbriefe oder andere Texte, werden, anders als in den früheren Bänden angegeben, aufgenommen, wenn keine Veröffentlichung der Texte existiert bzw. wenn sie zum Verständnis des Briefs, des Autors oder zur Beurteilung Gottscheds beitragen. Texte, die in der Leipziger Sammlung überliefert sind, aber eindeutig keinerlei Briefcharakter tragen, z. B. amtliche Erklärungen und nicht personenbezogene Gedichte, bleiben von der Veröffentlichung ausgeschlossen.

1 Wolfram Suchier: Alphabetisches Absenderregister zur Briefsammlung Gottscheds in der Universitätsbibliothek Leipzig. Berlin 1910-1912. 
Gewisse Probleme bereitete der Umgang mit Gottscheds amtlichem Briefwechsel, d.h. mit den Briefen, die Gottsched in seiner Position als Universitätslehrer, als Verwalter kursächsischer oder preußischer Stipendien, als Rektor und Dekan verfaßt hat. Briefe dieser Art befinden sich in größerer Zahl im Archiv der Leipziger Universität, darunter zahlreiche Schreiben, in denen der jeweilige Dekan den Kollegen der Philosophischen Fakultät ein Problem eröffnet und um ihr Votum bittet. Gottsched ist hier fast immer vertreten, entweder als Dekan in den Anschreiben oder unter den Voten der Fakultätsmitglieder. Sofern diese Briefe in der Sammlung der Universitätsbibliothek enthalten und von Suchier erfaßt worden sind, werden sie von uns gedruckt. Die im Universitätsarchiv überlieferten amtlichen Schreiben müssen zum großen Teil aus Kapazitätsgründen aus der Briefausgabe ausgeschlossen bleiben. Ihre Erschließung und die Bearbeitung des Bestandes bereitet Frau Dr. Caroline Köhler für eine separate Veröffentlichung vor.

\section{Briefkopf}

Die Schreiben werden in chronologischer Folge vorgelegt und innerhalb jedes Bandes fortlaufend numeriert. Die Kopfzeile bezeichnet Briefschreiber und Briefempfänger, mit Ausnahme von Gottsched immer mit vollem Namen, Absendeort und Datum. Für die Bezeichnung der Orte werden die Namen der Entstehungszeit in moderner Schreibweise angegeben. Erschlossene Angaben stehen in eckigen Klammern. Die in eckigen Klammern angegebenen Nummern nach dem Datum bezeichnen den letzten vorangegangenen und den nächstfolgenden Brief der jeweiligen Korrespondenz.

Unter dem Stichwort Überlieferung erfolgt der Hinweis auf sämtliche Überlieferungsträger: Original, Abschrift(en), Druck(e) und die besitzenden Institutionen (die am häufigsten vertretene Institution wird abgekürzt bezeichnet: Leipzig, UB für Universitätsbibliothek). Nach der Angabe der Signatur wird der Umfang des Briefes mitgeteilt. Gegebenenfalls wird auf Notizen wie z. B. Empfängervermerke hingewiesen, die sich auf dem Briefbogen befinden, aber nicht dem Brieftext selbst zugehören. Ältere Briefoder Blattzählungen, die auf einem großen Teil der in Leipzig überlieferten Briefe enthalten sind, werden nicht dokumentiert. Auch postalische Vermerke werden stillschweigend übergangen, sofern es sich um bloße Zahlenangaben handelt. 
Verzichtet wurde weiterhin auf alle Mitteilungen zum Format der Briefe, zu Siegelresten, zu den Wasserzeichen, zum Erhaltungszustand der Papiere u.a. Unter der Rubrik Drucke werden sämtliche Veröffentlichungen der Briefe registriert, bei Existenz des Originals jedoch nur vollständige Drucke. Ausnahmen bilden Briefpassagen, die Gottsched in eigenen Werken zitiert, und die Teildrucke in Theodor Wilhelm Danzels Werk Gottsched und seine Zeit. ${ }^{2}$ Die Ausnahme ist darin begründet, daß Danzels Werk bislang die Hauptquelle für die Kenntnis der Gottsched-Korrespondenz darstellt und in dieser Funktion in zahlreichen Publikationen zitiert wird. Durch den Nachweis sollte es möglich sein, nach Danzel zitierte Briefe ohne größeren Aufwand in unserer Ausgabe aufzufinden.

Soweit vorhanden, werden dem Druck unserer Ausgabe die Originalschreiben zugrundegelegt. Sind Stücke nur in Abschriften oder Drucken überliefert, werden diese als Textvorlage verwendet. Sollten mehrere Textzeugen vorliegen, wird im Briefkopf angegeben, welche Überlieferung als Druckvorlage dient. Ist das Originalschreiben vorhanden, werden Textvarianten der Abschrift oder des Drucks nicht vermerkt. Zusätzliche Angaben, mit denen die Aufnahme, die Datierung, der Absender oder der Adressat eines Briefes begründet wird, werden bei Bedarf unterhalb des Briefkopfes notiert. Auch die Regesten, die Inhaltsangaben zu den in französischer, italienischer und lateinischer Sprache verfaßten Briefen enthalten, werden unterhalb des Briefkopfes mitgeteilt.

\section{Textkonstitution}

Die Texte werden weitgehend diplomatisch getreu wiedergegeben. Einige Vereinfachungen und Vereinheitlichungen gibt es dennoch: Sätze werden immer mit Großbuchstaben begonnen. Auch Orts- und Personennamen werden unabhängig von der Vorlage groß geschrieben, ebenso die häufig abgekürzt verwendete Anrede Herr und alle Titelangaben der Anrede. Einige Sonderzeichen werden in Text überführt. Wenn z. B. ein diagonal durchgestrichener Kreis für das Wort „nicht" verwendet wird, schreiben wir das entsprechende Wort. Geminationsstriche über den Buchstaben $\mathrm{m}$ und $\mathrm{n}$ werden durch die Verdoppelung der Konsonanten dargestellt, verschliffene

2 Theodor Wilhelm Danzel: Gottsched und seine Zeit. Auszüge aus seinem Briefwechsel. Leipzig 1848 (mehrere Nachdrucke, zuletzt Eschborn 1998). 
Endungen für -en bzw. -em werden entsprechend dem jeweils erforderlichen Kasus wiedergegeben. Eindeutig ausgeschriebene Endungen werden nicht korrigiert. Ebensowenig werden die orthographischen Eigentümlichkeiten angetastet oder auch nur vermerkt. Bei sinnentstellten Wörtern wird, sofern möglich, eine Korrektur vorgenommen und im Textapparat nachgewiesen. Textpassagen, die im Original verlorengegangen sind, werden als Verluste in eckigen Klammern markiert. Ein Strich bezeichnet fehlende Buchstaben bzw. ein fehlendes Wort, zwei Striche zwei Wörter, drei Striche stehen für drei oder mehr verlorene Wörter. Sollte eine andere Überlieferung oder eine Konjektur durch den Bearbeiter angebracht sein, wird der Eingriff gekennzeichnet und im Textapparat nachgewiesen. Nicht eindeutig lesbare und deshalb nur als Konjektur des Bearbeiters zu verstehende Wörter werden in spitze Klammern gesetzt. Wenn eine Buchstabenfolge eine sinnvolle Konjektur nicht zuläßt, stehen in den spitzen Klammern analog zu den Textverlusten ein oder mehrere Striche.

Hervorhebungen (unterstrichen, fett, gesperrt, kursiv) werden kursiv wiedergegeben, Versalien werden im Druck beibehalten. Der unterschiedliche Schriftgebrauch für fremdsprachige Anteile in deutschen Briefen wird nicht dokumentiert. Abkürzungen werden im Erläuterungsapparat aufgelöst. Abkürzungen, von denen die Herausgeber meinen, daß sie aus dem Kontext verständlich oder noch heute gebräuchlich sind, werden nicht aufgelöst. Die Gliederung der Schreiben in neue Zeilen und Absätze folgt der Vorlage. Nur in der Anrede und in der Schlußformel werden die Zeilenumbrüche durch Schrägstriche gekennzeichnet. Postskripta werden nach den Briefen abgedruckt, auch wenn die Verfasser die Nachschriften am Rande der vorderen Briefseiten notieren. Adressen werden am Schluß der Briefe abgedruckt.

\section{Textapparat}

Der Textapparat ist wegen der relativ einfachen Überlieferungssituation eher schmal. Im Textapparat werden Streichungen, Korrekturen und Ergänzungen der Briefautoren dokumentiert, sofern sie von sachlicher Relevanz sind. Einfache Textersetzungen durch Streichung werden durch Ziffern angezeigt, die die Folge der Streichung und Ersetzung nachvollziehbar machen sollen. Alle weiteren Auskünfte sind verbalisiert, die von den Bearbeitern stammenden Herausgebermitteilungen sind kursiv, die Brieftexte 
recte wiedergegeben. Beruht die Edition auf Abschriften oder Drucken, werden relevante Lesarten der anderen, nicht als Druckvorlage verwendeten Überlieferungen im textkritischen Apparat angegeben. Im Textapparat werden die Siglen A für Abschrift und D für Druck verwendet.

\section{Erläuterungsapparat}

Der Erläuterungsapparat enthält Informationen zu den erwähnten Personen, Titelangaben der aufgeführten Publikationen, exakte Stellennachweise für die im Text erwähnten literarischen Sachverhalte, Nachweise von Zitaten sowie Worterklärungen und erklärt die in den Briefen angesprochenen Umstände unter Anführung von Quellen oder Sekundärliteratur. In den Fällen, in denen es nicht möglich war, entsprechende Informationen zu ermitteln, steht im Apparat der Vermerk „nicht ermittelt“. Sofern Personen im Deutschen Biographischen Index (3. Auflage, München 2004), in den entsprechenden anderen nationalen Indizes oder bei Wikipedia verzeichnet sind, entfallen Angaben zur Herkunft der Information. Für weiterreichende Informationen werden Quellen- bzw. Literaturhinweise angeführt. Die Titelangaben beruhen nach Möglichkeit auf Autopsie, die Titel werden gekürzt wiedergegeben, Ziel ist die zweifelsfreie Identifizierbarkeit der genannten Literatur. Der Nachweis entfällt, wenn Korrespondenten Kleinschrifttum schicken oder von Gottsched empfangen haben, für dessen Ermittlung alle Anhaltspunkte fehlen.

Zitate aus antiken Schriften werden durch Angabe der Stellen und ohne Ausgabe nachgewiesen, sofern aus dem Brieftext nicht die Benutzung einer konkreten Edition nahegelegt wird.

Für den Nachweis von Schriften Gottscheds wird zumeist auf die von Phillip M. Mitchell erarbeitete Bibliographie und die dort vergebene Nummer verwiesen. ${ }^{3}$ Textnachweise erfolgen soweit möglich anhand der Ausgewählten Werke Gottscheds, ${ }^{4}$ die dank vorzüglicher Textapparate die Wiederauffindbarkeit von Zitaten in den einzelnen Auflagen erlauben. Gedichte Gottscheds werden, soweit möglich, nach der separaten ersten Ausgabe der

${ }^{3}$ Johann Christoph Gottsched: Ausgewählte Werke. Hrsg. von Phillip M. Mitchell. Band 12: Gottsched-Bibliographie. Berlin; New York 1987.

${ }^{4}$ Johann Christoph Gottsched: Ausgewählte Werke. 12 Bände. Berlin; New York 1968-1995. 
Texte angegeben. Andernfalls erfolgt ein Hinweis auf den Druck in der zweibändigen Gedichtsammlung von 1751.

Erläuterungen zu den Personen enthalten die Lebensdaten und die wichtigsten beruflichen Stationen, darüber hinaus Hinweise, die zum Verständnis des entsprechenden Brieftextes erforderlich sind. Sofern die betreffende Person zu den Korrespondenten Gottscheds gehört, werden diese Angaben durch den Hinweis „Korrespondent“ ergänzt. Weitere Informationen über diese Personen vermittelt das bio-bibliographische Korrespondentenverzeichnis, sofern deren Briefe im vorliegenden Band enthalten sind. Für andere Korrespondenten verweisen wir auf die früheren Bände unserer Ausgabe und das kumulierte Verzeichnis, das über die Website des Verlags Walter de Gruyter GmbH bzw. der Editionsstelle erreichbar ist.

Sofern Literaturtitel nicht standardisiert nachgewiesen, sondern im Kontext einer Erläuterung erwähnt werden, werden sie kursiviert. Die in den Erläuterungen häufiger erwähnte Literatur wird nur mit Kurztiteln angegeben. Der vollständige Titel ist im Abkürzungsverzeichnis zu finden. Verweise auf noch nicht edierte Briefe erfolgen durch die Angabe des Datums.

\section{Verzeichnisse}

Der Band enthält Verzeichnisse der Absender, der Absendeorte, der Fundorte und der abgekürzt zitierten Literatur. Das bio-bibliographische Korrespondentenverzeichnis umfaßt Lebensdaten, Angaben zu den wichtigsten biographischen Stationen und zur Anzahl der Briefe. Der Umfang der angegebenen Sekundärliteratur variiert nach dem Stand der Forschungsliteratur. Für bekannte Personen genügte der Verweis auf eine Bibliographie bzw. auf wichtige Titel. Bei weitgehend unbekannten Personen wurden sämtliche Titel aufgeführt, denen Angaben zur betreffenden Person entnommen werden konnten. Wenn eine Person im Deutschen Biographischen Index enthalten ist, findet sich am Ende der Literaturangaben der Vermerk DBI. Die dort integrierte Literatur wird von uns nicht eigens aufgeführt. Dem Korrespondentenverzeichnis folgen Personen-, Orts- und Schriftenverzeichnisse. Bibelstellen sind in das Schriftenverzeichnis integriert und dort unter dem Stichwort Bibel zu finden. Die Schriften Gottscheds sind separat aufgeführt: Abweichend vom allgemeinen Verzeichnis werden sie in Anlehnung an die in der Gottsched-Bibliographie von Mitchell verwende- 
ten Numerierung in chronologischer Reihenfolge registriert. Schriften, die in der Bibliographie nicht verzeichnet sind, werden am Ende des jeweiligen Jahres ohne Vergabe einer Nummer aufgeführt. Die Werke der Luise Adelgunde Victorie Gottsched sind in dieses Verzeichnis integriert. Auch diese Verzeichnisse sind kumuliert über die Website von Verlag und Editionsstelle abrufbar.

Bearbeiter der Briefe:

Korrespondenten A-E sowie Briefwechsel Reiffstein und Thomasius: Caroline Köhler; Korrespondenten F-N, Briefe von Johann Nathanael Reichel sowie Gottsched an Zanotti und an Querini: Rüdiger Otto; Korrespondenten O-T: Michael Schlott; Korrespondenten W, Briefwechsel Flottwell und Scheyb sowie Brief Querini an Gottsched: Franziska Menzel 
